\title{
Mitteilung aus der Redaktion
}

\section{Information from the Editors}

Liebe Leserinnen und Leser,

für die Zukunft ist ein erweitertes Angebot der Kasuistiken vorgesehen:

Wir möchten erreichen, dass Kasuistiken eingereicht werden, zu deren Verlauf es offene Fragen gibt. Das kann bedeuten, dass es in der Nachschau Fragen gibt, aber auch, dass die Behandlung noch nicht abgeschlossen ist oder einen Punkt erreicht hat, an dem ein neuer Schritt nötig ist.

Voraussetzung für die Einsendung ist, dass der Fall mit einer Suchterkrankung zu tun hat und die formalen Kriterien einer Kasuistik erfüllt:

Es kann sich dabei um Kurzkasuistiken handeln, die sich mit besonderen therapeutischen Problemstellungen beschäftigen. Diese können einerseits diagnostische Fragen (etwa Komorbidität, spezielle diagnostische Verfahren) und deren Konsequenzen für Therapieansätze (etwa differenzielle Indikation, sequenzielles Vorgehen in einer Intervallbehandlung) betreffen. Andererseits können Kasuistiken von besonderem Wert sein, die spezielle Therapieansätze oder Modifikationen bestehender Therapieansätze (etwa Therapie bei minderbegabten Abhängigen) schildern. Schließlich können Kasuistiken relevant sein, die besondere epidemiologische Konstellationen oder Risikosituationen beleuchten (etwa Kinder alkoholkranker Eltern).

Folgende Manuskriptanforderungen sind notwendig:

1. Die Titelseite sollte den Titel der Kurzkasuistik, die beteiligten Autoren (Vorname, Name, Standort) sowie Namen und Adresse des federführenden Autors beinhalten.
2. Die Kurzkasuistiken sollen folgende Gliederung aufweisen: Problemstellung (maximal eine Manuskriptseite), Kasuistik (maximal zwei Manuskriptseiten), Diskussion (maximal zwei Manuskriptseiten), Literatur (maximal 5 Literaturzitate).

3. Eine Manuskriptseite darf nicht mehr als 30 Zeilen à 60 Zeichen umfassen.

Die Zeitschrift Suchttherapie wird dann für ausgewählte Kasuistiken drei Experten aus den Beiräten der Zeitschrift, dem Zentrum für Interdisziplinäre Suchtforschung Hamburg, der Deutschen Gesellschaft für Suchtmedizin oder der Deutschen Gesellschaft für Suchtpsychologie zu Rate ziehen, die dann ihrerseits ein Gutachten zu der jeweiligen Kasuistik abgeben werden. Die Gesamtbetrachtung wird dann in einem der folgenden Hefte erscheinen und/oder im Internet, und zwar im öffentlichen $\mathrm{Zu}$ gangsbereich von Thieme-connect.

Bitte senden Sie Ihre Kasuistik als Ausdruck und/oder als Wordkompatible Datei an:

Georg Farnbacher

Zentrum für Interdisziplinäre Suchtforschung der

Universität Hamburg

c/o Zentrum für psychosoziale Medizin, Psychiatrie und Psychotherapie im UKE

Martinistr. 52

20246 Hamburg

E-mail: farnbacher@uke.uni-hamburg.de 